\title{
Essential oils utility implications in symptomatic Burning Mouth Syndrome
}

\author{
PATANO Assunta ${ }^{1,+}$, DI VENERE Daniela, ${ }^{1+}$ CECI Sabino ${ }^{1}$, BERATE Pula ${ }^{2}$, CANDREA Sebastiann ${ }^{3,+}$, \\ BABTAN Anida-Maria ${ }^{3,}{ }^{*}$, AZZOLLINI Daniela ${ }^{1}$, PIRAS Fabio ${ }^{1}$, CURATOLI Luigi ${ }^{4}$, \\ CORRIERO Alberto ${ }^{5}$, VALENTE Francesco ${ }^{1}$, MAGGIORE Maria Elena ${ }^{1}$, \\ MANCINI Antonio ${ }^{1}$, GIOVANNIELLO Delia ${ }^{6}$, NUCCI Ludovica ${ }^{7}$, ELIA Rossella ${ }^{8}$, \\ SIRBU Adina ${ }^{9,}{ }^{+}$, FEURDEAN Claudia ${ }^{3,},{ }^{*}$, GALDERISI Andrea10, CARDARELLI Fillippo ${ }^{1,+}$
}

Editor: Constantin Munteanu, Romanian Association of Balneology, office@,bioclima.ro

Reviewers: Silisteanu Sinziana Calina and Rotariu Mariana

*Corresponding author: BABTAN, Anida-Maria, E-mail: anidamaria.babtan@gmail.com

1. Department of Interdisciplinary Medicine (D.I.M), University of Medicine “Aldo Moro”, Bari, Italy

2. Privat clinic, Allias Vure, Rruga, Tirane, Albania

3. Department of Oral Rehabilitation, University of Medicine and Pharmacy "Iuliu Hatieganu”, Cluj-Napoca, Romania

4. Department Neurosciences \& Sensory Organs \& Musculoskeletal system. University of Bari “Aldo Moro”, Bari, Italy

5. Unit of Anesthesia and Resuscitation, Department of Emergencies and Organ Transplantations, "Aldo Moro"

University, Bari, Italy

6. Hospital A.O.S.G. Moscati, Contrada Amoretta, Avellino (AV), Italy

7. Multidisciplinary Department of Medical-Surgical and Dental Specialties, University of Campania "Luigi Vanvitelli",

Naples, Italy

8. Bari University “Aldo Moro”, place Giulio Cesare, Bari, Italy

9. Department of Oral Health, University of Medicine and Pharmacy "Iuliu Hatieganu”, Cluj-Napoca, Romania

10. Università degli Studi di Napoli Federico II, Napoli, Italy

\section{Abstract}

Introduction. Burning mouth syndrome (BMS) is a clinical condition characterized by the presence of chronic pain in absence of clinically visible lesions of the oral mucosa. The etiology is uncertain and the therapeutic strategies still controversial. The objective of this prospective study is to analyze the efficacy of essential oils-based mouthwashes in the therapy of BMS.

Material and method. This study included 16 patients affected by BMS who were treated with essential oils-based mouthwashes and glucose solution on alternated days for 30 days. Symptomatology was evaluated after 15, 30 and 90 days.

Results and discussions. A the end of the treatment, most of the patients $(67 \%)$ referred an improvement of symptoms up to complete remission in 90 days.

Conclusions. Based on this study, essential oils-based mouthwashes could represent a valid aid in the treatment of BMS. Further studies are necessary in order to identify effective and standardized therapeutic protocols.

Keywords: Burning Mouth Syndrome; oral rinse; essential oils; therapeutic strategies,

\section{INTRODUCTION}

Burning mouth syndrome (BMS) is a clinical condition characterized by burning symptoms and chronic pain, affecting the oral mucosa, not referable to objective alterations of the physiological muco-membranous architecture of tissues of the oral cavity $(1,2)$. It is a difficult pathology for both the operator and the patient due to absence of a clear etiological definition and welldefined and effective therapeutic plans (3).

The most controversial aspect of BMS is represented by the presence of a single symptom (stomatopyrosis) which can potentially manifest itself as many clinical conditions such as nutritional deficiencies, hormonal imbalances, diabetes (4). These aspects lead to the differentiation of BMS in two types:

- Primary, characterized by the absence of associated clinical conditions

- Secondary, characterized by the presence of associated clinical conditions

The prevalence of BMS in the general population is between 2.5 and $5 \%$ (5). In most cases, BMS affects females (F:M ratio $=7: 1)$ in the menopausal and postmenopausal age (between 55 and 75 years), while it is very rare in young people and never observed in children.

In order to make the clinical classification more understandable, in 1989, Lamey and Lewis suggested a classification, in which three clinical forms of BMS were present, based upon variation of symptoms during the day (6):

1. Absence of burning sensation at awakening, onset of symptoms during the morning and exacerbation in the evening (often associated with nutritional deficiencies);

2. Continuous symptoms throughout the day (associated with anxiety); 
3. Intermittent symptoms with days of remission and days of exacerbation (associated with allergic states).

Burning sometimes referred to as a sense of scalding or tingling, is always present. Although it can be located in any area of the oral cavity, it always arises bilaterally and affects in most cases the tip of the tongue and the anterior $2 / 3$, the hard palate and the lower lip while rarely, the floor of the mouth and the oropharynx (7).

The burning symptom has a moderate intensity but sometimes can be quite severe. The values reported by the Visual Analogic Scale are between 5 and 8 . The course is chronic; it lasts at least 4-6 months up to 12 years. It is often accompanied by a sensation of xerostomia (50-60\% of cases) and by hypogeusia and dysgeusia, referring to a persistent perception of bitter or metallic taste $(30 \%$ of cases).

Regarding the etiological hypotheses, local and systemic factors can be distinguished. Among the local etiological factors there are:

- Vices (bruxism, grinding, biting of the lips and / or cheeks);

- Dental treatments;

- Infectious agents (Candida Albicans, Enterobacter, Klebsiella);

- Allergic reactions to particular substances (Mercury, Nickel Sulphate, Methyl-Methacrylate Monomers)

- Salivary changes:

- quantitative: decrease in salivary flow;

- qualitative: alteration of salivary concentrations of proteins (mucins) and salts (potassium and sulphates)

- Peripheral neuropathies (8).

On the other hand, systemic factors include:

- Menopause;

- Vitamin (Vitamin B and folic acid) and mineral (Iron and Zinc) nutritional deficiencies;

- Diabetes;

- Psycological disorders: anxiety, depression, hypochondria, cancer-phobia, alexithymia, neurosis and psychoasthenia).

The frustrating aspect of this clinical condition is the difficulty in identifying the agent triggering the symptoms and consequently identifying an appropriate therapeutic protocol. However, it seems that certain molecules play an interesting role in the symptomatic treatment of BMS.

Typically, prescribed therapy consists of (9):

- Clonazepam: this benzodiazepine not only has an anxiolytic effect but also acts at the level of sensory receptors producing a moderate analgesic effect;

- Salivary substitutes;

- Vitamin supplements (with zinc);

- Benzydamine HCL 3\% solution: it is a mild anesthetic (it is assumed that the patient with BMS has a peripheral neuropathy due to epithelial atrophy making the nerve endings more superficial with consequent amplification of symptoms even for mild stimuli);

- Topical antihistaminics;

- Antidepressants;

- Capsaicin: acts on substance C (an important molecule in neurotransmission). Although not extensively evaluated, capsaicin seems to be able to block the burning receptors with a homeopathic mechanism;

- Alpha-lipoic acid: an essential oil that acts as a mitochondrial coenzyme and that takes part in various phases of the Krebs cycle with a double effect

Antioxidant effect, meaning the capacity of the enzymatic or not enzymatic system of limiting the harmful effect of reactive chemical species (RCS). These molecules have in common a marked tendency to react. They are physiologically produced; however, an imbalance between production and elimination determines a condition of oxidative stress, which can occur in physiological conditions, for example in case of inflammation, or pathological conditions, if it persists over time. In this case, it can be correlated with numerous pathophysiological and pathological states such as senescence, diabetes mellitus type II, atherosclerosis, Alzheimer disease), cancer, COPD, hepatopathies and more (10-12).

It was shown that in vitro, alpha lipoic acid presents 4 antioxidants activities: the ability to cross-link metals, to eliminate reactive oxygen, to regenerate endogenous antioxidants and to repair oxidative damage (13).

The neuroprotective effect is given to the ability to cross the blood brain barrier and increase the energy availability, hence its use in multiple sclerosis and BMS, since considered by some authors a peripheral neuropathy with an unknown etiology (14). On the basis of these considerations, a prospective study was carried out to assess whether and to what extent the treatment with essential oils-based (menthol, eucalyptol, thymol) mouthwash was able to reduce the symptoms of patients suffering from BMS.

\section{Material and Method}

Sixteen patients with symptoms compatible with BMS were recruited. Patients underwent an odontostomatological examination, blood routines (complete blood count, glycemia, iron, transferrin, vitamin B12, folate), intraoral tongue brushing for the evaluation of any mycosis. Based on their symptoms, only patients who did not present other possible causes were included in the study.

The inclusion criteria were: males and females ( $>45 y$ years old), with BMS symptoms, no ongoing drug treatment (antiretroviral, antihistamine, anticholinergic, narcotic, anti-inflammatory drugs), no other treatments for BMS. Those who refused to continue the treatment for 90 days were excluded.

The present prospective clinical study was based in 
University of "Aldo Moro", Bari (Italy), in full accordance with ethical principles, including the World Medical Association Declaration of Helsinki and the additional requirements of Italian law. Furthermore, the University of Bari, Italy, classified the study to be exempt from ethical review as it carries only negligible risk and involves the use of existing data that contains only nonidentifiable data about human beings. Informed consent was obtained from the subjects involved in the study. Written informed consent has been obtained from the patient to publish this paper.

The essential BMS was diagnosed in 12 of these subjects, and in 4 individuals with no characteristic symptoms. They were aged between 44 and 78 years. Using a singleblind control method, for 3 months every other day patients were asked to use:

a) Essential oils-based mouthwash (3 oral rinses lasting $60 \mathrm{sec} /$ day);

b) Sweetened saline solution (same dosage and method of administration).

Symptoms were assessed by compiling a visual-analog scale (V.A.S.) (Fig. 1) after 15 days, 30 days and 90 days.

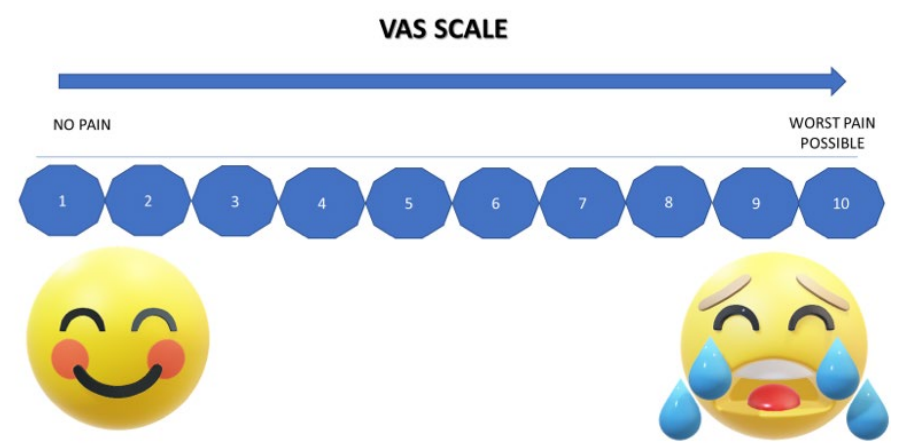

Fig. 1. VAS scale in symptomatic pain assessment.

\section{Results}

This study was performed on 16 patients with BMS with the mean age (Fig. 3) of 61.3 years ranged from 44 to 78 years, including 9 women (56\%) and 7 men (44\%) (Fig. 2).

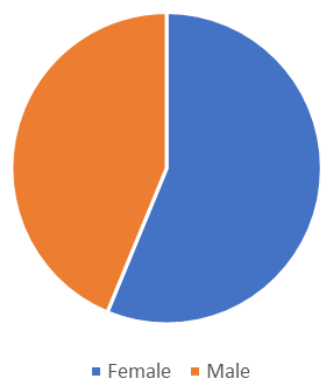

Fig. 2. Gender repartition of the included subjects.

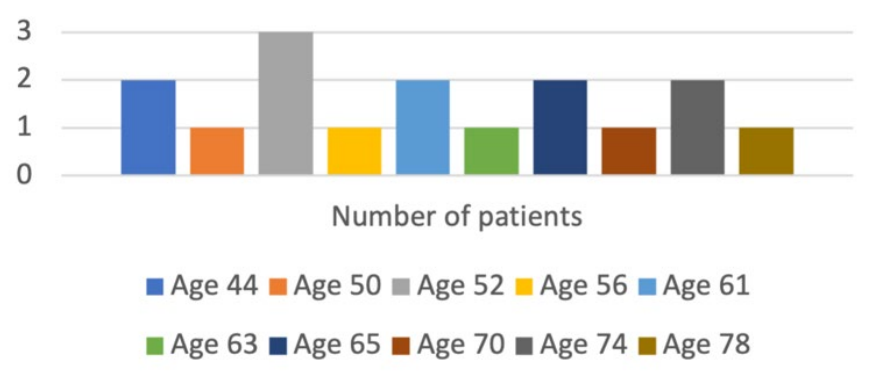

Fig. 3. Age range of the included subjects

- 67\% of patients (Fig. 4) reported a reduction of symptoms after administration of essential oils-based mouthwash (3 oral rinses lasting $60 \mathrm{sec} /$ day) followed by a remission of pain with relief of the patient;

- $13 \%$ of subjects reported no difference in symptomatology;

- $5 \%$ of patients reported an increased level of pain when taking both the control solution and the essential oilsbased mouthwash.

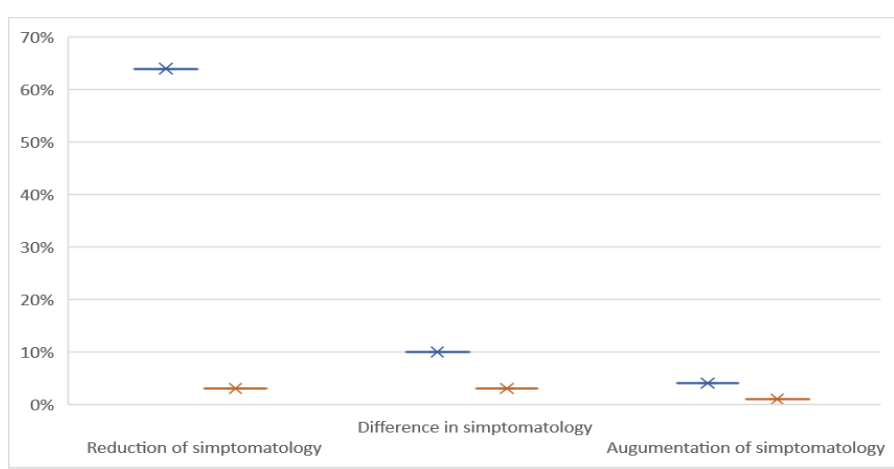

Fig. 4. Simtomatology assessment after treatment.

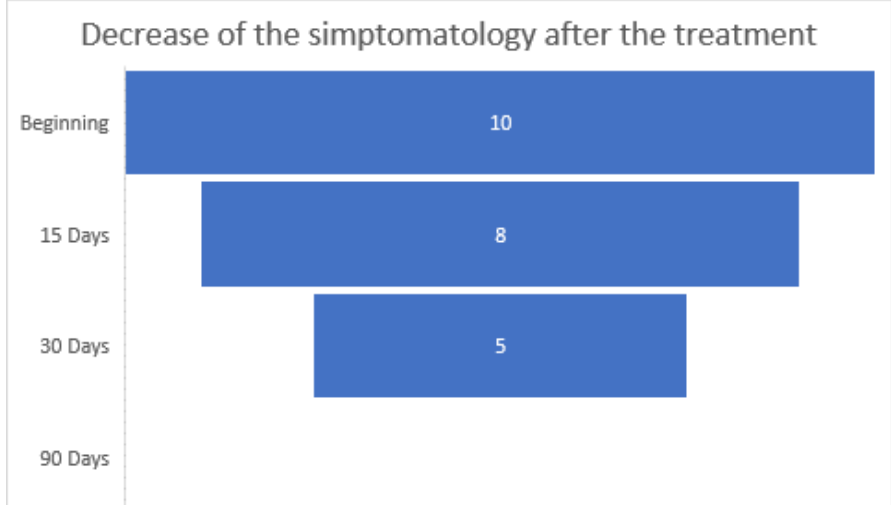

Fig. 5. VAS scale during BMS treatment.

Regarding the trend analysis of the symptoms, a reduction in VAS was reported by patients (Fig. 5), on average, from a value of 10 before treatment to a value of 8 after 2 weeks, until a VAS value of 5 after 30 days of treatment. Patients underwent a 90-days follow-up in which the total absence of symptoms was reported. 


\section{Discussion}

The BMS still represents a particularly frustrating pathology for both the patient and the clinician today. The real problem is to define the etiological factors that play the key role in the pathogenesis and therefore to establish an effective therapeutic protocol (15).

It is believed that in the evaluation of the patient with BMS it is essential to investigate the psycho-social status, establishing a relationship ad personam that can be corroborated through optimal counseling between the patient and the clinician. The histological picture of BMS it is completely devoid of pathognomonic signs; sometimes the presence of sporadic hyphae of Candida Albicans can be found (16). The diagnosis of this syndrome is mainly based on an accurate medical history concerning the psycho-social status of the patient, the reported symptoms and the total absence of stomatognathic clinical signs. In any case, it is important to reassure the patient of the absolute benignity of her clinical condition. The therapeutic approaches used so far seem to alleviate the symptoms but they are often based on personal empirical experiences and not always reproducible on a large scale (17).

The results of the present study show that essential oilsbased mouthwashes are useful in reducing the symptoms of patients with BMS. This benefit may be linked to the anti-inflammatory, antioxidant, immune-stimulating and antimicrobial properties of essential oils (18-20).

Furthermore, the pharmacological activity of essential oils on the central nervous system is well known and therefore, they are already used as complementary therapy in subjects suffering from symptoms such as pain, convulsions and cognitive impairment by oral intake, inhalation or aromatherapy (21-24). This action on the central nervous system could be useful in BMS, for which a neuropathic origin has been hypothesized, characterized by dysfunctional cranial nerves (25). In addition, the anxiolytic, antidepressant and sedative effect of essential oils plays an important role in patients with BMS $(26,27)$. Actually, subjects affected by this clinical condition tend to be more apprehensive, depressed and introverted.

Limitations of the study include low sample size and the pattern that does not grant a high level of evidence (such as the evaluation of non-specific placebo effect). Finally, the essential oils-based mouthwash effects have only been tested on the short term ( 90 days).

\section{Conclusion}

Based on the research conducted, it is possible to reach the conclusion that the action of essential oils seems to produce positive effects on symptoms control. The pilot study is preliminary and needs further evaluation. However, the first results show a partial improvement of symptoms in patients treated with essential oils-based mouthwash.

\section{Conflicts of interest}

The authors declare no conflicts of interest.

\section{Author contributions.}

Patano Assunta and Di Venere Daniela contributed equally as first co-author of this paper. Sirbu Adina and Cardarelli Fillippo contributed equally as last co-author of this paper.

\section{References}

1. Marino R, Previdi M, Colangelo G, Spadai F.Burning Mouth Syndrome: the role of the contact hypersensitivity. Giorn It Allergol Immunol Clin. 2005; 15:51-63.

2. Hanna R, Dalvi S, Benedicenti S, Amaroli A, Sălăgean T, Pop ID, Todea D, Bordea IR. Photobiomodulation Therapy in Oral Mucositis and Potentially Malignant Oral Lesions: A Therapy Towards the Future. Cancers (Basel). 2020 Jul 18;12(7): 1949. doi: 10.3390/cancers12071949. PMID: 32708390; PMCID: PMC7409159.

3. Chiniforush N, Pourhajibagher M, Parker S, Benedicenti S, Bahador A, Sălăgean T, Bordea IR. The Effect of Antimicrobial Photodynamic Therapy Using Chlorophyllin-Phycocyanin Mixture on Enterococcus faecalis: The Influence of Different Light Sources. Applied Sciences. 2020; 10(12): 4290. https://doi.org/10.3390/app10124290

4. Signorini L, Ballini A, Arrigoni R, De Leonardis F, Saini R, Cantore S, De Vito D, Coscia MF, Dipalma G, Santacroce L, Inchingolo F. Evaluation of a nutraceutical product with probiotics, vitamin d, plus banaba leaf extracts (Lagerstroemia speciosa) in glycemic control. Endocr Metab Immune Disord Drug Targets. 2020; Nov 8. doi: 10.2174/1871530320666201109115415.

5. Locker D, Grushka M. Prevalence of oral and facial pain and disconfort: preliminary results of a mail survery. Community Dent Oral Epidemiol. 1987;15:169-7.

6. Lamey PJ, Lamb AB Prospective study of aetiological factors in burning mouth syndrome. $\mathrm{Br}$ Med J 1988; 296:1243-6.

7. Lipton JA, Ship JA, Larach-Robinson D.-Estimated prevalence and distribution of orofacial pain in the United States. J Am Dent Assoc. 1993; 124:115-21. 
8. Glick D, Ben Aryeh H, Gutman D, et al. Realtion between glossodynia and salivary flow rate and content. Int J Oral Surg 1976; 5:161-5.

9. Sellers EM, Khanna JM. Anxiolytics, hypnotics and sedatives. In: Kalant H, Roschlau W. Editors principles of pharmacology. Toronto BC Decker Publisher; 1989. p 255-64.

10. Ziegler D, Gries FA. L'acido alfa-lipoico nel trattamento della neuropatia periferica e cardiaca da diabete. Diabetes 1997; Sep; 46 Suppl 2: S62.

11. Packer L; Tritschler HJ; Wessel K.-Neuroprotezione dell'antiossidante metabolico acido alfa-lipoico. Free Radic Biol Med. 1997; 22(1-2):359-78.

12. Bustamante J, Lodge JK, Marcocci L, Tritschler HJ, Packer L, Rihn BH-L'acido alfa-lipoico nel metabolismo e nelle malattie epatiche. Free Radic Biol Med 1998 Apr; 24(6):1023-39.

13. Biewenga GP, Haenen GR, Bast A. La farmacologia dell'antiossidante acido lipoico. Gen Pharmacol 1997 Sep; 29(3):315-31.

14. Patton LL, Siegel MA, Benoliel R, De Laat A. Management of burning mouth syndrome: systematic review and management recommendations. Oral Surg Oral Med Oral Pathol Oral Radiol Endod. 2007 Mar; 103 Suppl: S39.e113.

15. Inchingolo F, Marrelli M, Annibali S, Cristalli MP, Dipalma G, Inchingolo AD, Palladino A, Inchingolo AM, Gargari M, Tatullo M. Influence of endodontic treatment on systemic oxidative stress. Int J Med Sci. 2013 Dec 6;11(1):1-6. doi: 10.7150/ijms.6663.

16. Marrelli M, Tatullo M, Dipalma G, Inchingolo F. Oral infection by Staphylococcus aureus in patients affected by White Sponge Nevus: a description of two cases occurred in the same family. Int J Med Sci. 2012; 9(1): 47-50.

17. Signorini L, Inchingolo AD, Santacroce L, Xhajanka E, Altini V, Bordea IR, Dipalma G, Cantore S, Inchingolo F. Efficacy of combined sea salt based oral rinse with xylitol in improving healing process and oral hygiene among diabetic population after oral surgery. J Biol Regul Homeost Agents. 2020 Jul-Aug; 34(4): 1617-1622. doi: 10.23812/20-418-L. PMID: 32909424.

18. Valdivieso-Ugarte, Magdalena et al. "Antimicrobial, Antioxidant, and Immunomodulatory Properties of Essential Oils: A Systematic Review." Nutrients. 2019; vol. 11, 11.

19. Bakkali F, Averbeck S, Averbeck D, Idaomar M. Biological effects of essential oils - A review. Food and Chemical Toxicology. 2008; 46(2): 446-475.

20. Anastasiou C., Buchbauer G. Essential Oils as Immunomodulators: Some Examples. Open Chem. 2017; 15:352-370.

21. De Sousa DP. Analgesic-like activity of essential oils constituents. Molecules. 2011; 16:2233-2252.

22. Inchingolo F, Dipalma G, Cirulli N, Cantore S, Saini RS, Altini V, Santacroce L, Ballini A, Saini R. Microbiological results of improvement in periodontal condition by administration of oral probiotics. J Biol Regul Homeost Agents 2018; 32.

23. De Almeida RN, Agra MF, Maior FNS, de Sousa DP. Essential oils and their constituents, anticonvulsant activity. Molecules. 2011; 16:27262742.

24. Wang ZJ, Heinbockel T. Essential Oils and Their Constituents Targeting the GABAergic System and Sodium Channels as Treatment of Neurological Diseases. Molecules 2018; 2; 23(5):1061.

25. Kanchan R, Patil KR, Sathawane RS. Burning mouth syndrome: Clinical dilemma? J Oral Med Oral Radiol. 2008; 20:129-33.

26. Malcolm BJ, Tallian K. Essential oil of lavender in anxiety disorders: Ready for prime time? Ment Health Clin. 2018; 26; 7(4):147-155.

27. Mubeen K, Neera Ohri BD. Burning mouth syndrome-an enigma. Int J Odontostomat 2011; 5(1):23-27. and PRM Research Journal. 2021;12(1):46-52. 